\title{
Hanford Site Regional Population - 2010 Census
}

\author{
EL Hamilton \\ SF Snyder
}

August 2011

Pacific Northwest

NATIONAL LABORATORY

Proudly Operated by Battelle Since 1965 


\title{
DISCLAIMER
}

This report was prepared as an account of work sponsored by an agency of the United States Government. Neither the United States Government nor any agency thereof, nor Battelle Memorial Institute, nor any of their employees, makes any warranty, express or implied, or assumes any legal liability or responsibility for the accuracy, completeness, or usefulness of any information, apparatus, product, or process disclosed, or represents that its use would not infringe privately owned rights. Reference herein to any specific commercial product, process, or service by trade name, trademark, manufacturer, or otherwise does not necessarily constitute or imply its endorsement, recommendation, or favoring by the United States Government or any agency thereof, or Battelle Memorial Institute. The views and opinions of authors expressed herein do not necessarily state or reflect those of the United States Government or any agency thereof.

\author{
PACIFIC NORTHWEST NATIONAL LABORATORY \\ operated by \\ BATTELLE \\ for the \\ UNITED STATES DEPARTMENT OF ENERGY \\ under Contract DE-ACO5-76RL01830
}

Printed in the United States of America
Available to DOE and DOE contractors from the
Office of Scientific and Technical Information,
P.O. Box 62, Oak Ridge, TN 37831-0062;
ph: (865) 576-8401
fax: $(865) 5765728$
email: reports@adonis.osti.gov

\author{
Available to the public from the National Technical Information Service, \\ U.S. Department of Commerce, 5285 Port Royal Rd., Springfield, VA 22161 \\ ph: (800) 553-6847 \\ fax: (703) 605-6900 \\ email: orders@nits.fedworld.gov \\ online ordering: http://www.ntis.gov/ordering.htm
}




\title{
Hanford Site Regional Population - 2010 Census
}

\author{
EL Hamilton \\ SF Snyder
}

August 2011

Prepared for the U.S. Department of Energy under Contract DE-AC05-76RL01830

Pacific Northwest National Laboratory

Richland, Washington 99352 



\section{Summary}

The U.S. Department of Energy (DOE) conducts radiological operations in south-central Washington State. Population dose estimates must be performed to provide a measure of the impact from site radiological releases. Results of the U.S. 2010 Census were used to determine counts and distributions for the residential population located within 50 miles of several operating areas of the Hanford Site.

Year 2010 was the first census year that a 50-mile population of a Hanford Site operational area exceeded the half-million mark. 



\section{Contents}

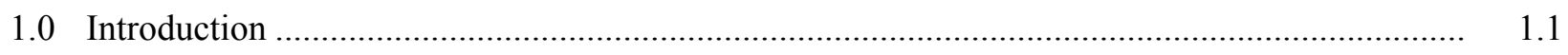

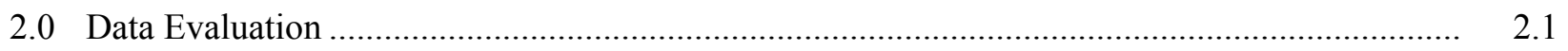

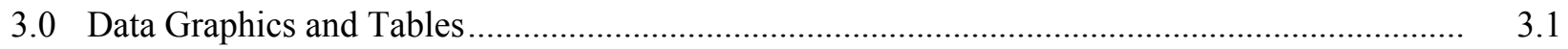

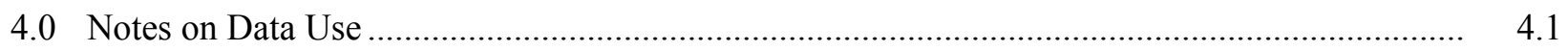

5.0 Multi-year Population Summary …........................................................................... 5.1

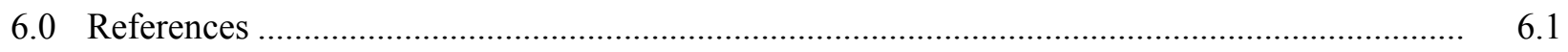

\section{Figures}

1.1200 HMS 50-mile Region and County Overlay................................................................ 1.1

2.1 Centroid Locations for 50-mile Population Distributions...................................................... 2.2

2.2 100K Centroid 50-mile Region and Census Block Overlay ..................................................... 2.3

2.3200 Area HMS Centroid 50-mile Region and Census Block Overlay....................................... 2.4

2.4300 Area Centroid 50-mile Region and Census Block Overlay ............................................. 2.5

2.5 400 Area Centroid 50-mile Region and Census Block Overlay .............................................. 2.6

2.6 WTP Centroid 50-mile Region and Census Block Overlay ................................................. 2.7

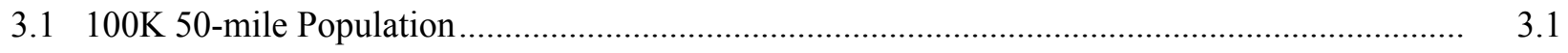

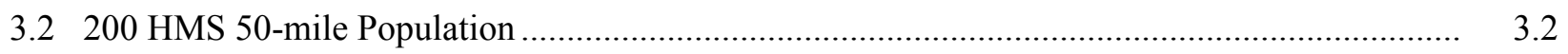

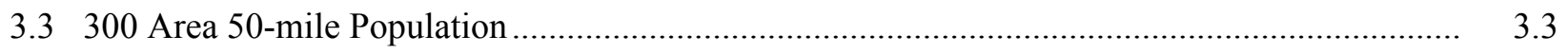

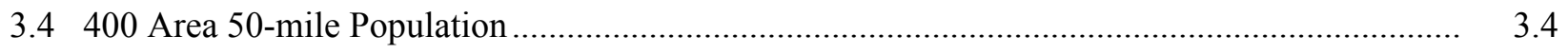

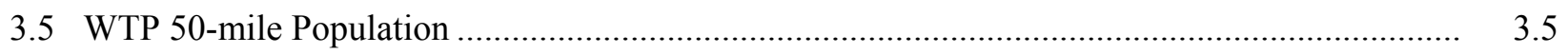

\section{Tables}

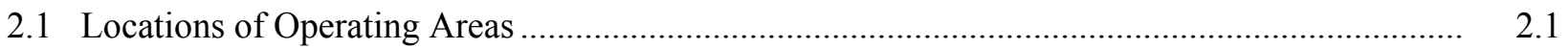

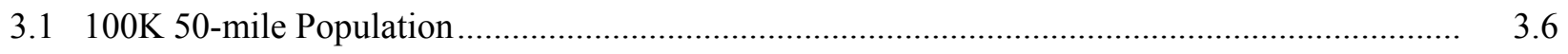

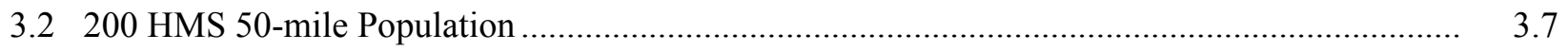

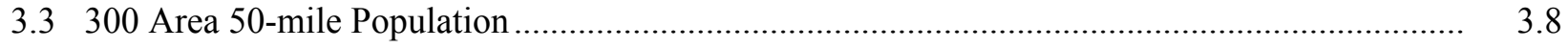

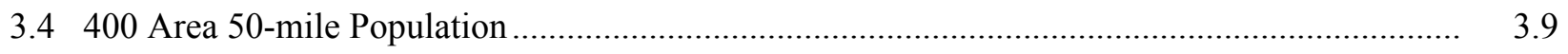

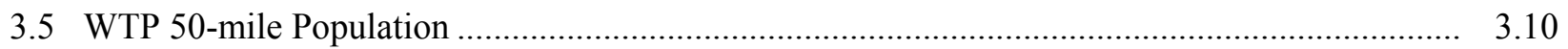

5.1 50-mile Populations - 1990, 2000, and 2010 Census'........................................................... 5.1 



\subsection{Introduction}

The newly available 2010 U.S. Census data were used to update 50 -mile residential population data relative to five major operating areas of the Hanford Site in Washington State. This information updates Elliott et al. (2004), which provided data from the year 2000 Census. The 1990 Census data evaluation was published in Beck et al. (1991). For 2010, the population within 50-miles is provided for a radial grid of 160 segments centered on each of five operating areas. Figure 1.1 indicates the Hanford Site boundary and county borders with the 50-mile grid centered on the 200 Area Hanford Meteorological Station (HMS). Morrow and Umatilla Counties are in Oregon. All other counties are in Washington.

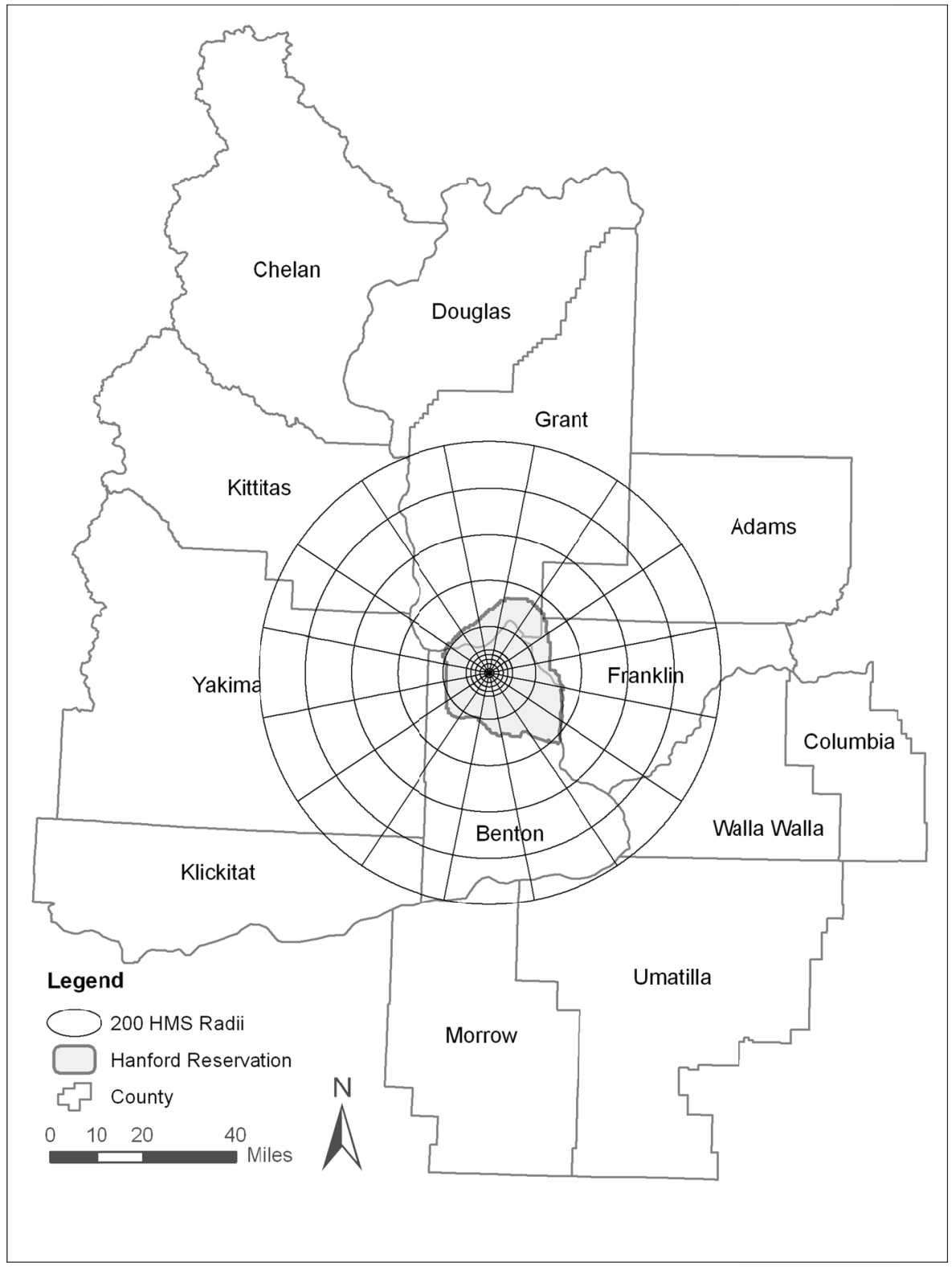

Figure 1.1. 200 HMS 50-mile Region and County Overlay 



\subsection{Data Evaluation}

Geographic information system (GIS) software, ArcInfo 9.3.1 (ESRI 2009), was used to create direction- and distance-specific circular grids centered on each of five Hanford Site locations: $100 \mathrm{~K}$ Area, 200 Area Hanford Meteorological Station (HMS) located between the 200-West and 200-East Areas, 300 Area, 400 Area, and the Hanford Tank Waste Treatment and Immobilization Plant (WTP) site located east of the 200-East Area (Table 2.1; Figure 2.1). The grids contained concentric circles with radii of $1,2,3,4,5,10,20,30,40$, and 50 miles, expanding from the point of one of five Hanford locations. The 16 directional sectors of the grid were created using the four cardinal directions $(\mathrm{N}, \mathrm{E}, \mathrm{S}, \mathrm{W})$, the four ordinal directions (NE, SE, SW, NW), and the eight half-wind directions (NNE, ENE, ESE, SSE, SSW, WSW, WNW, NNW). The 10 concentric distance circles were then combined with the 16 directional sectors to create a circular grid with 160 sections (Figure 2.2 through Figure 2.6).

Table 2.1. Locations of Operating Areas

\begin{tabular}{lcc}
\hline Operating Area & Longitude & Latitude \\
\hline 100K Area & -119.578 & 46.657 \\
200 HMS & -119.599 & 46.563 \\
300 Area & -119.286 & 46.364 \\
400 Area & -119.360 & 46.430 \\
WTP & -119.521 & 46.556 \\
\hline
\end{tabular}

In order to determine the population totals for each circular grid section, Census 2010 Tiger/Line block-level shapefiles containing Census 2010 Redistricting population totals (Census 2010a, 2010b, 2010c) were brought into the GIS. The area was calculated for each block and the population was assumed to be evenly distributed within that area. Then the block-level boundaries were intersected with the circular grids. The area was again calculated for the newly intersected blocks. To calculate a new population for each of the 160 grid segments, the areas for the block portions within a grid were multiplied by their respective original average census block population densities, then summed and rounded to the nearest whole number. For example, if a circular grid section contained $40 \%$ of the original area of a block, then it was assumed that the section also contained $40 \%$ of the block's original population. This procedure was repeated for grids centered at each location of interest to create the 50 -mile $(80-\mathrm{km})$ population distribution based on the 2010 census data. See Figure 2.2 through Figure 2.6 for census block overlays with the 50-mile radial grid. 


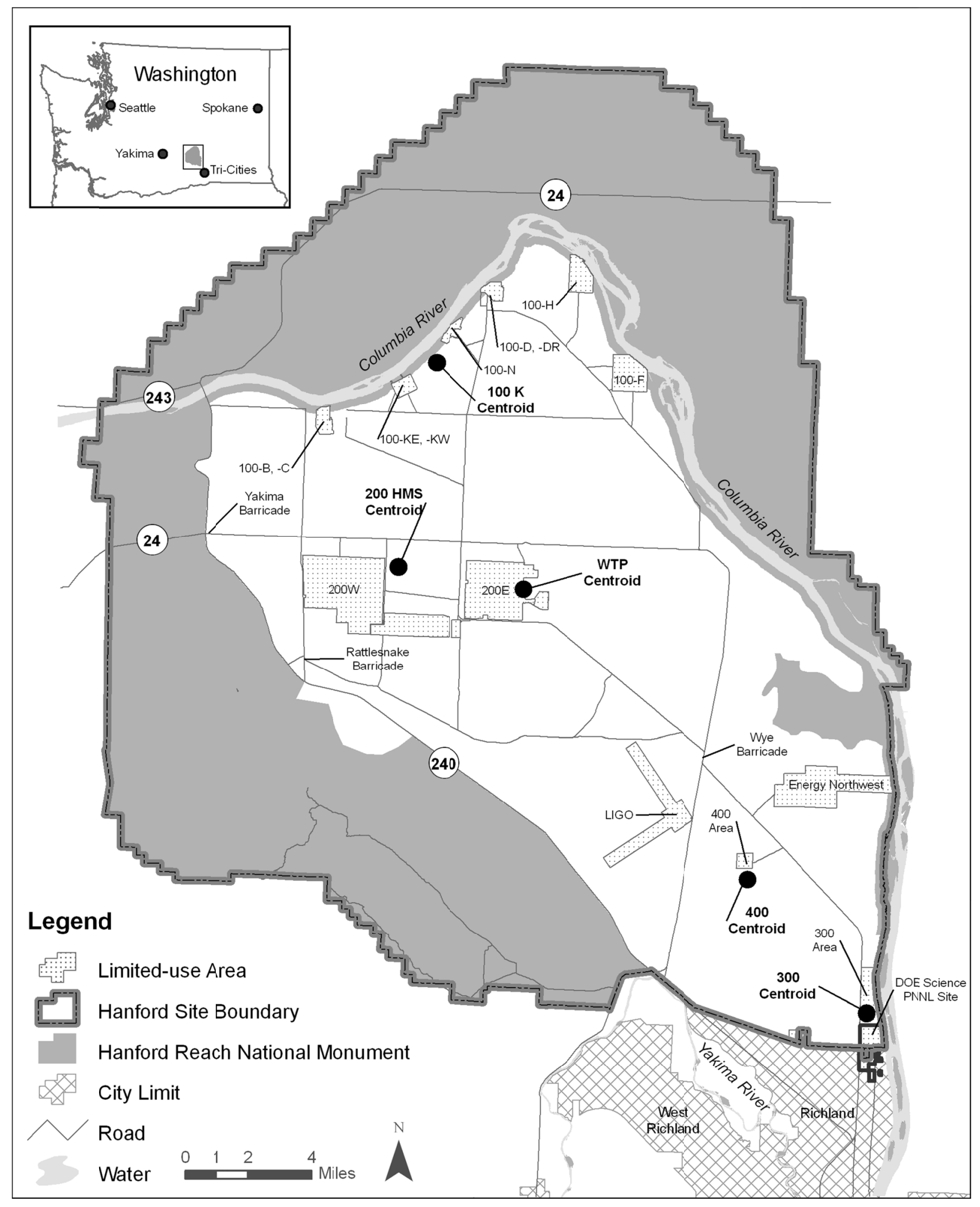

Figure 2.1. Centroid Locations for 50-mile Population Distributions 


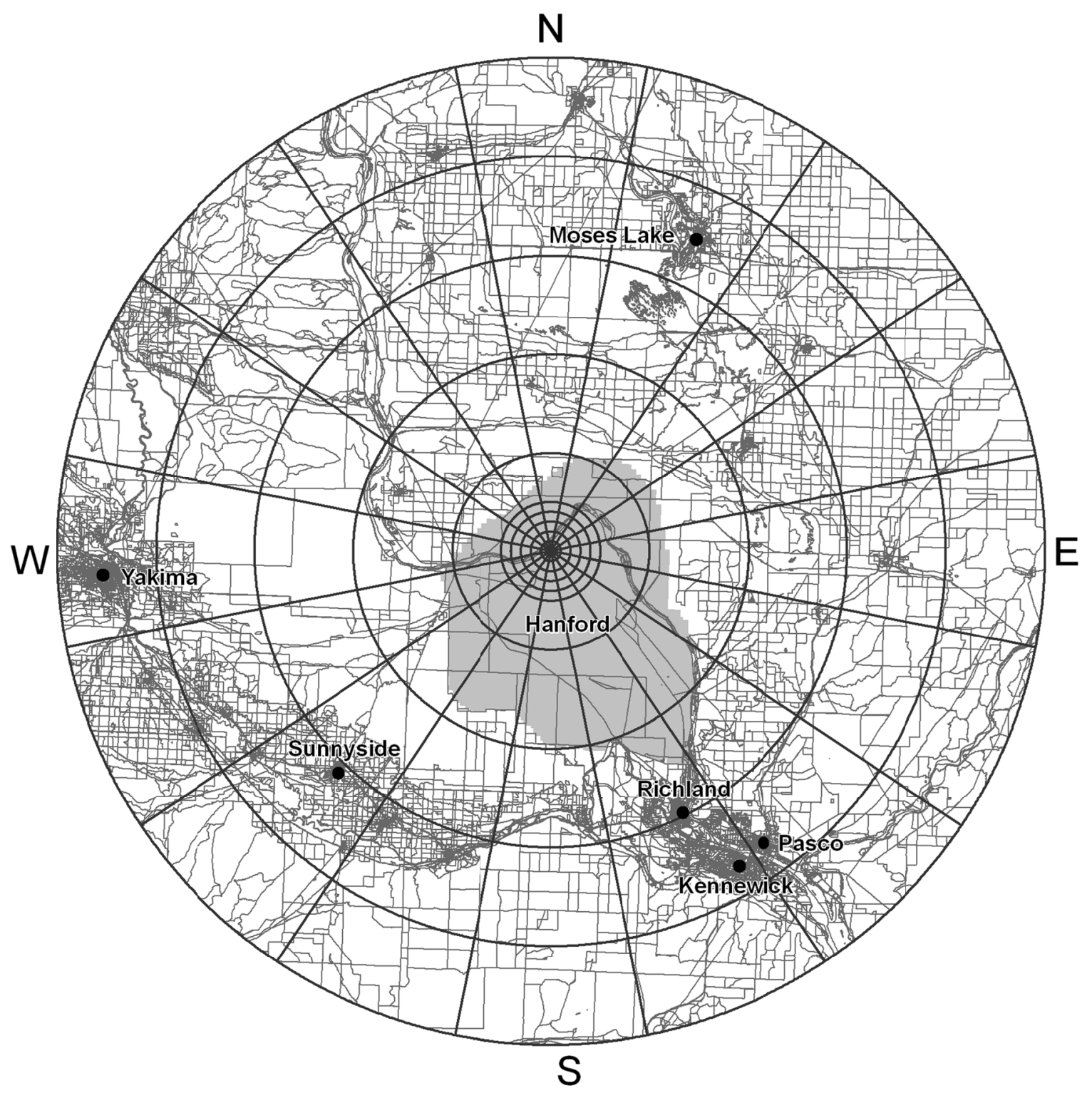

Figure 2.2. 100K Centroid 50-mile Region and Census Block Overlay 


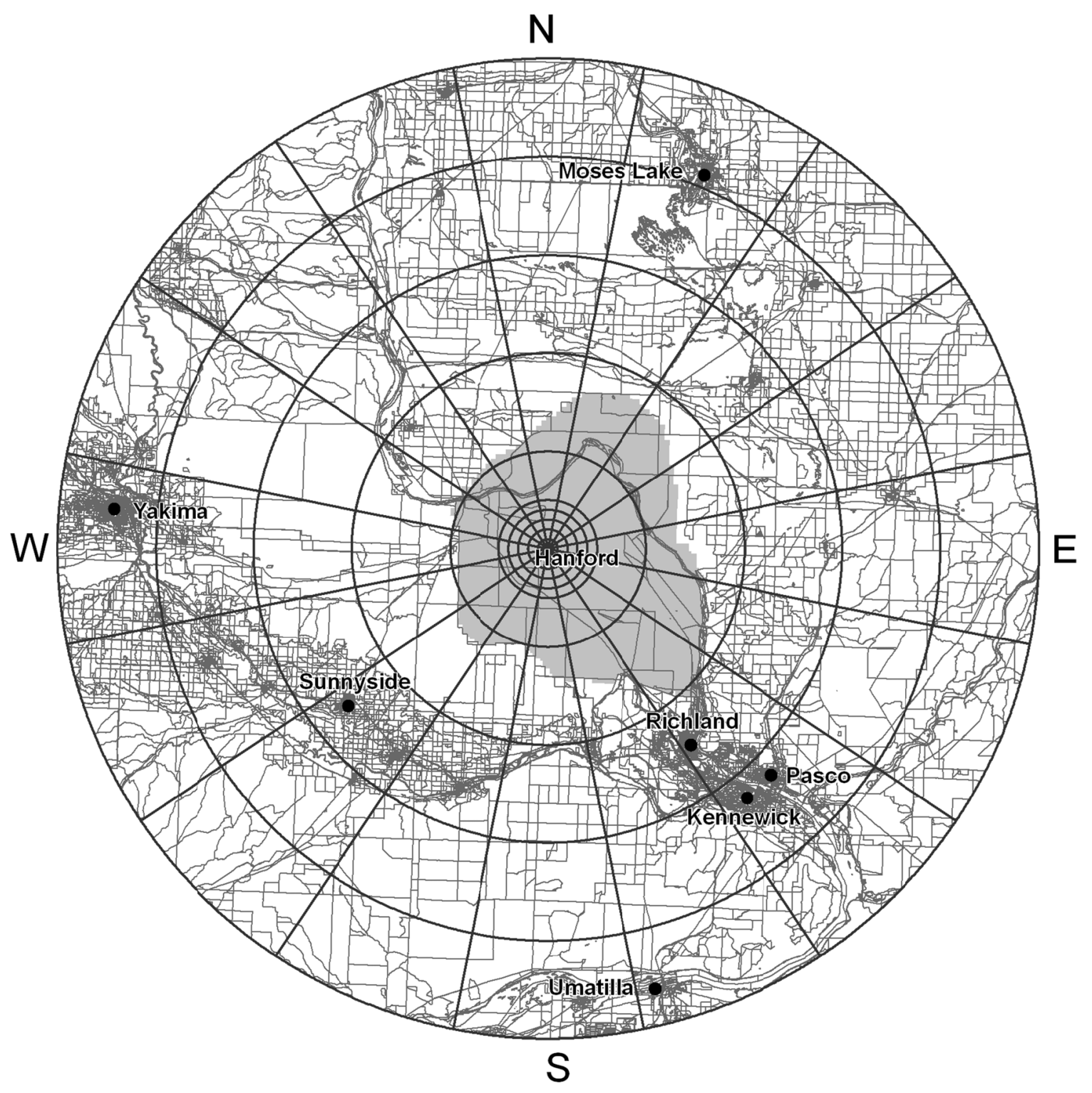

Figure 2.3. 200 Area HMS Centroid 50-mile Region and Census Block Overlay 


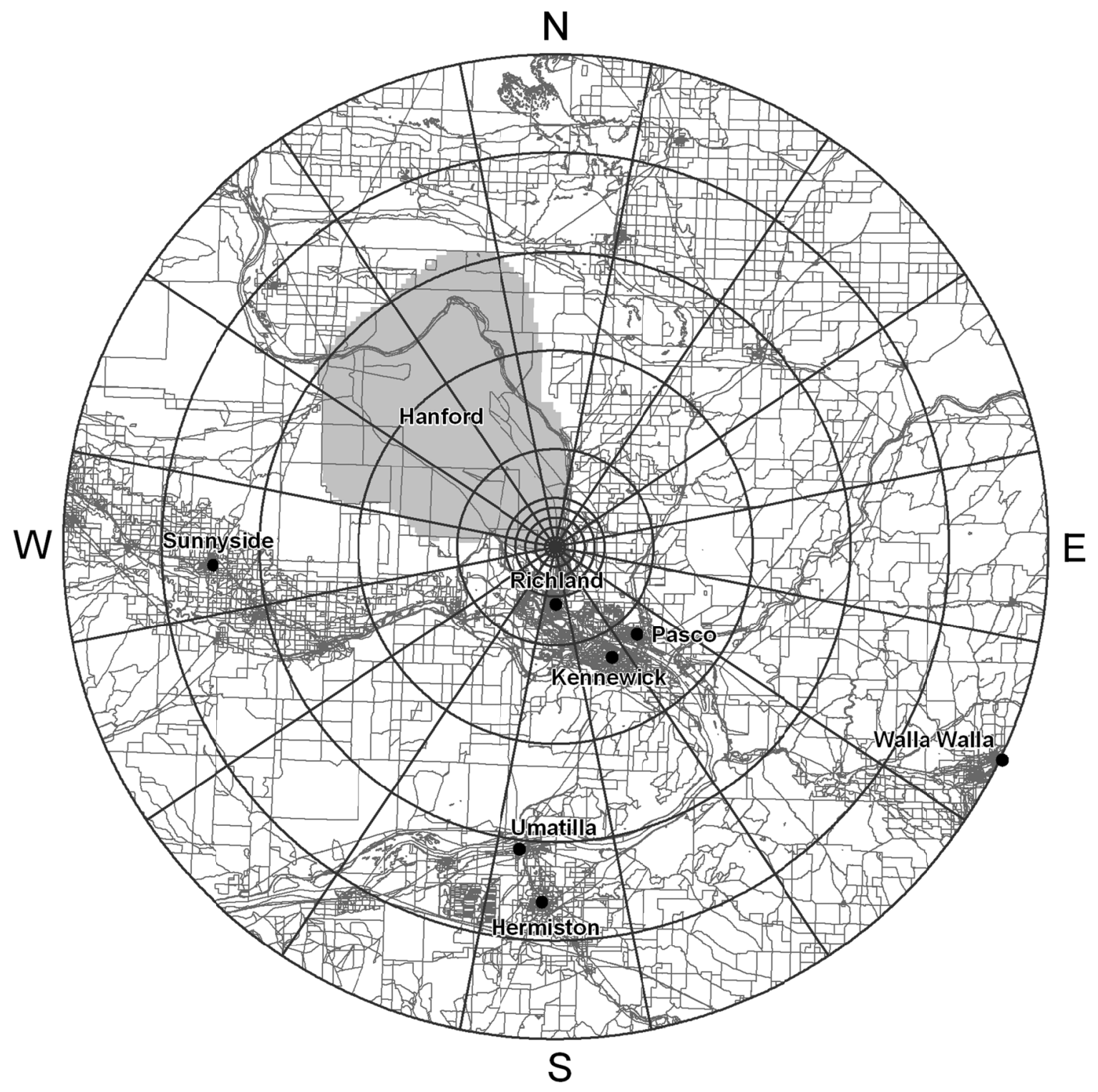

Figure 2.4. 300 Area Centroid 50-mile Region and Census Block Overlay 


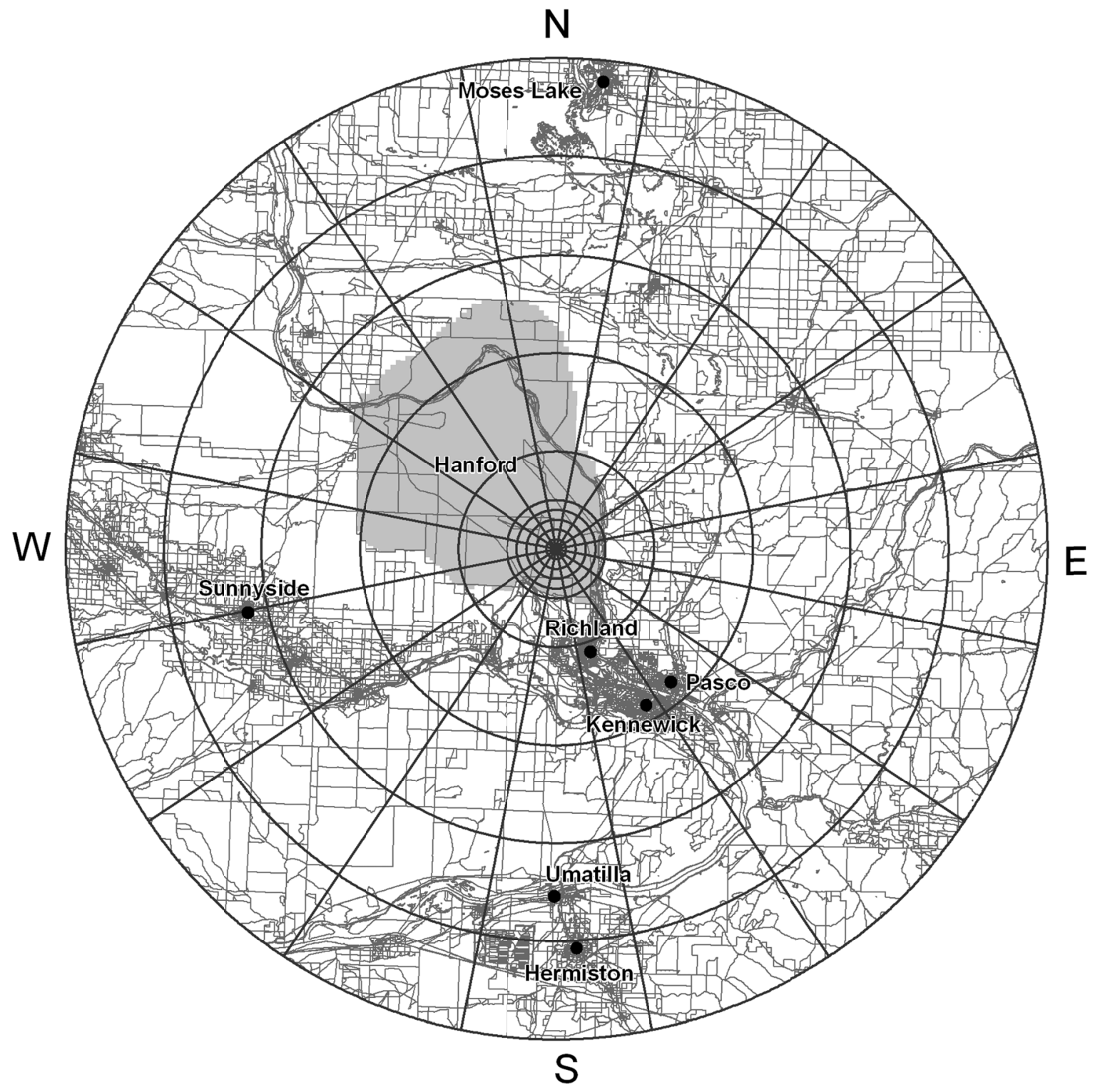

Figure 2.5. 400 Area Centroid 50-mile Region and Census Block Overlay 


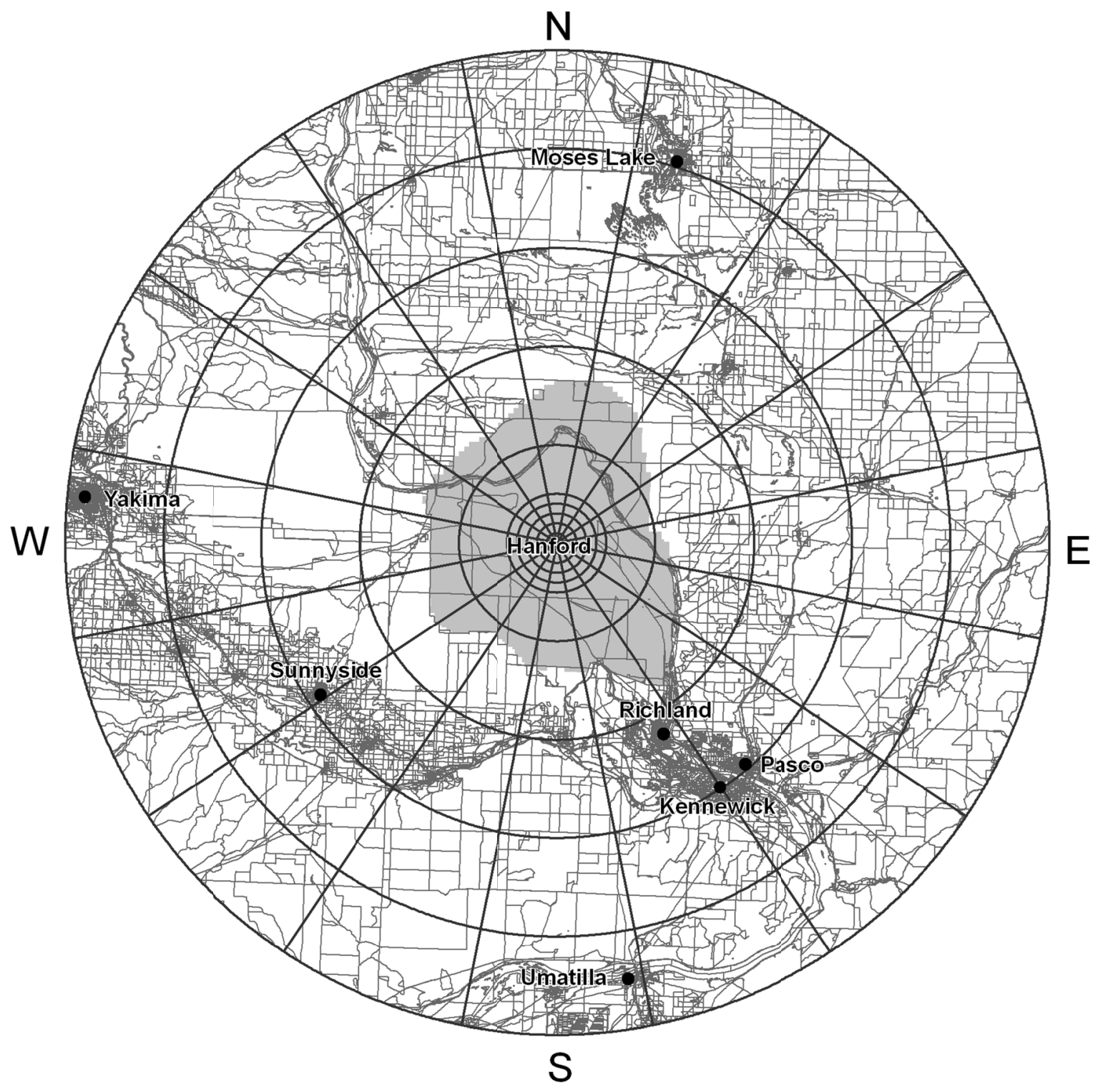

Figure 2.6. WTP Centroid 50-mile Region and Census Block Overlay 



\subsection{Data Graphics and Tables}

The data resulting from the evaluation described in Section 2.0 are presented here in graphic and tabular form. The user can then format the population distributions as needed for a particular application. Figure 3.1 through Figure 3.5 indicate the 5-10 mile and additional 10 mile-increment populations in the larger grid, and the 1-mile increment populations in the smaller 0-5 mile inset. Grid directions follow the compass rose with the top center sectors as North. Table 3.1 through Table 3.5 present the data in tabular form.

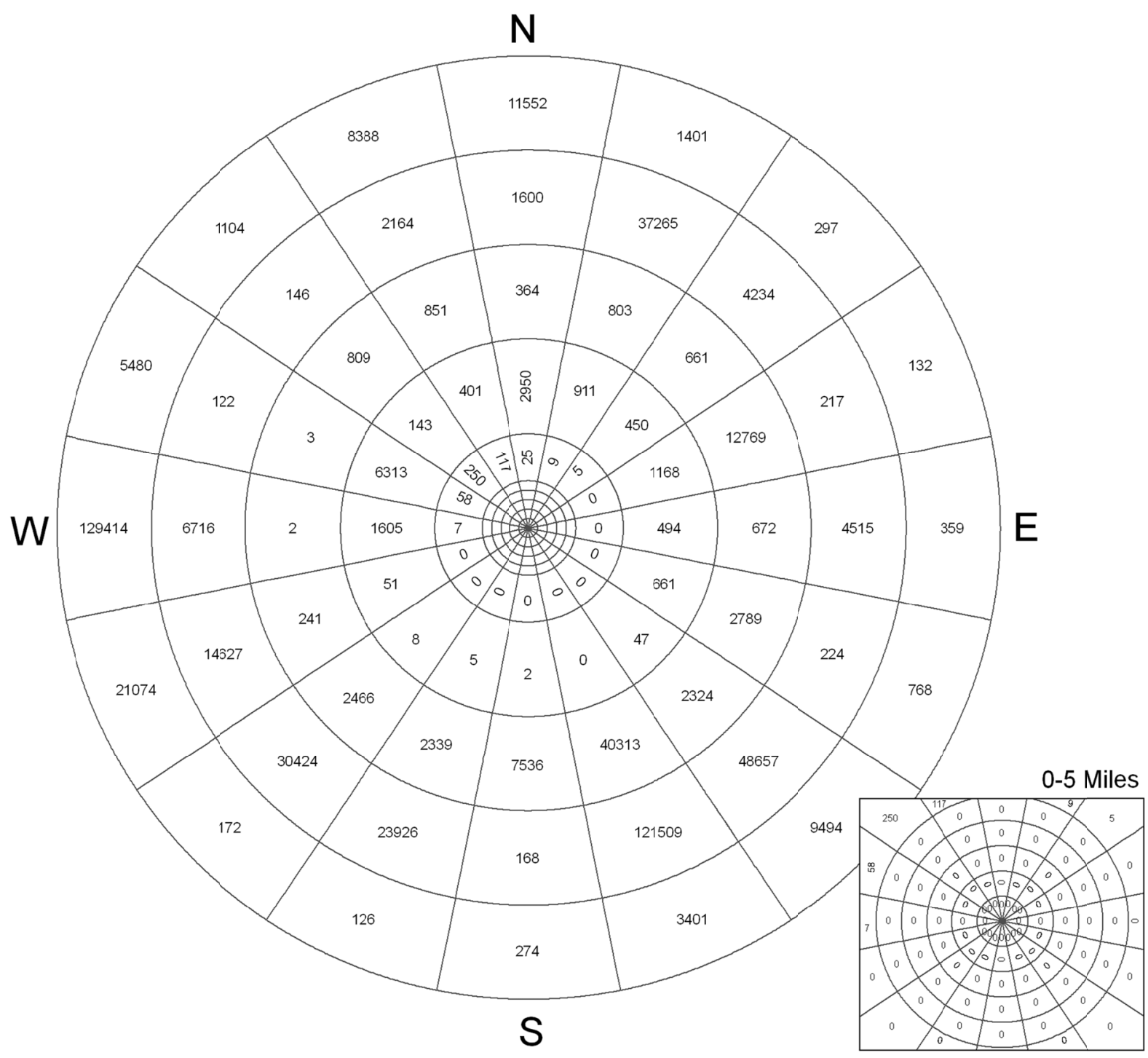

Figure 3.1. 100K 50-mile Population 


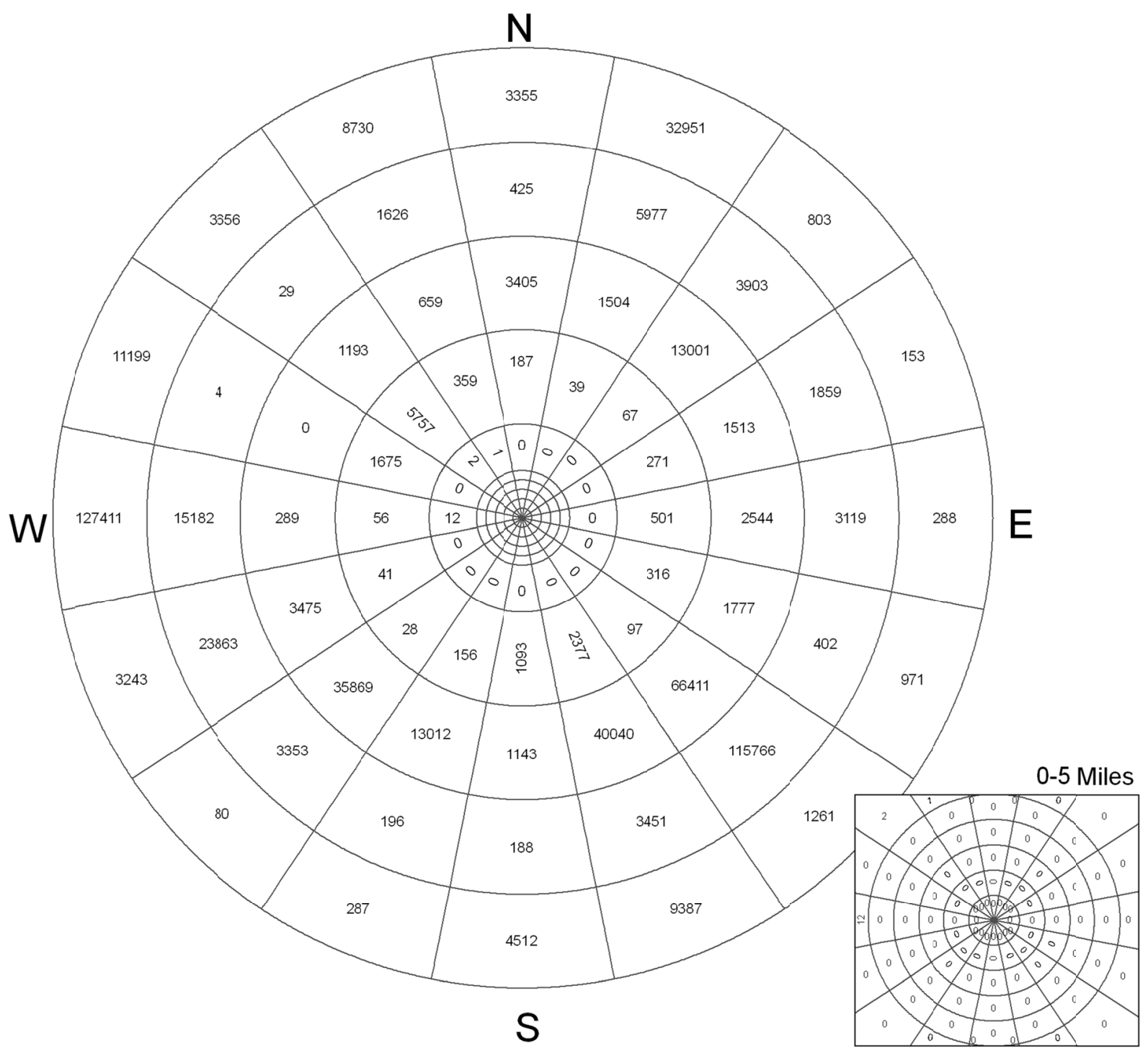

Figure 3.2. 200 HMS 50-mile Population 


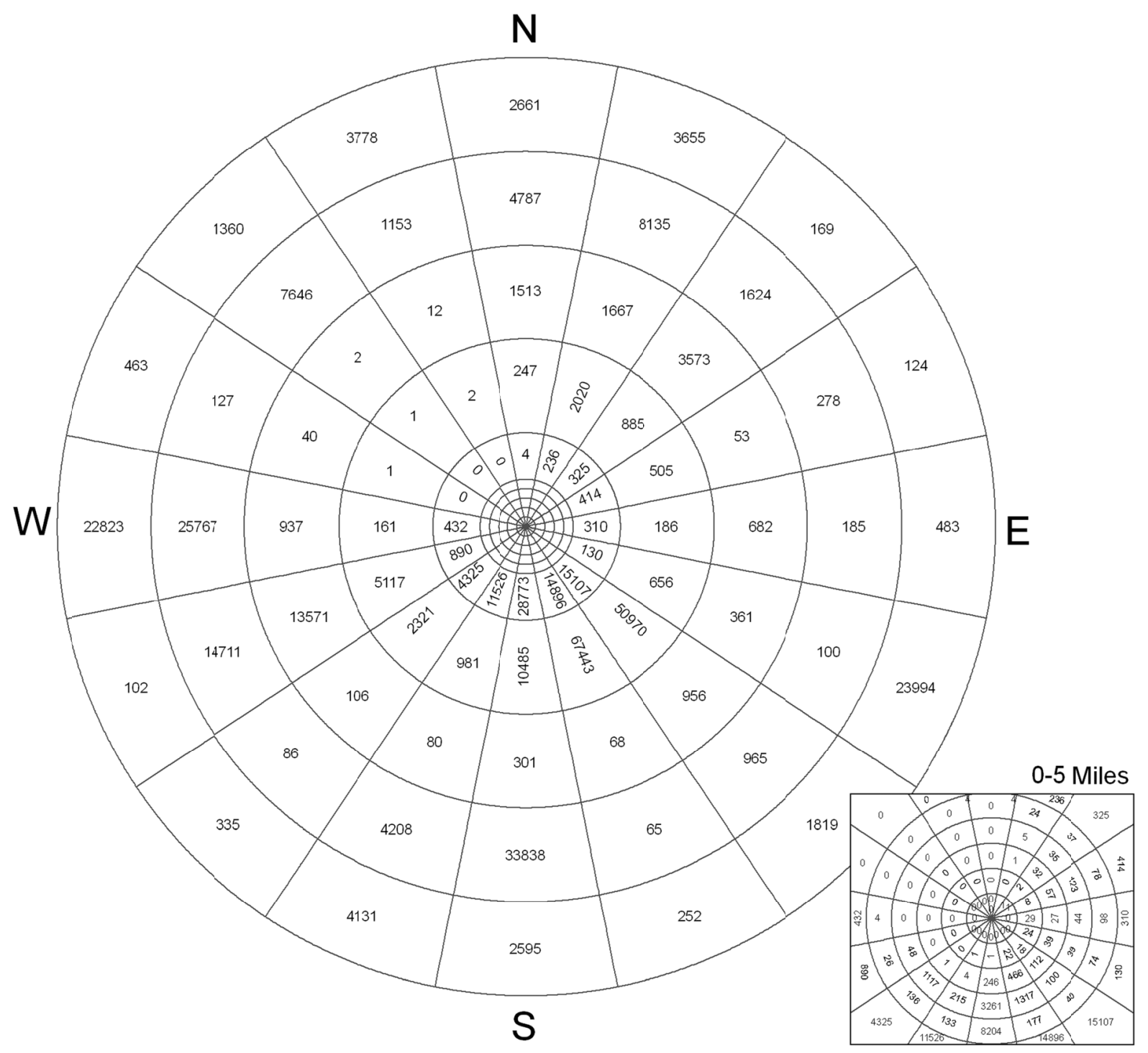

Figure 3.3. 300 Area 50-mile Population 


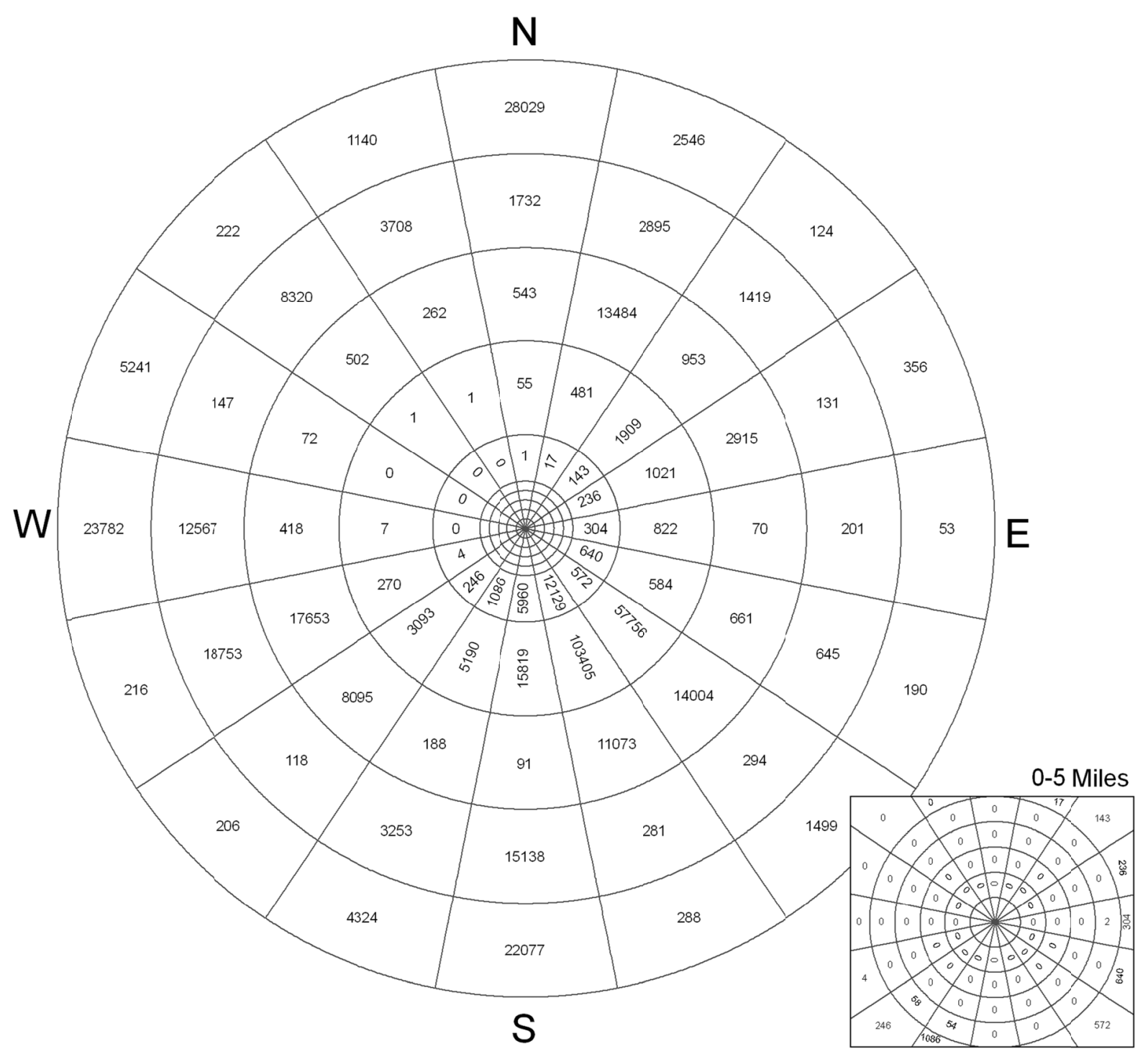

Figure 3.4. 400 Area 50-mile Population 


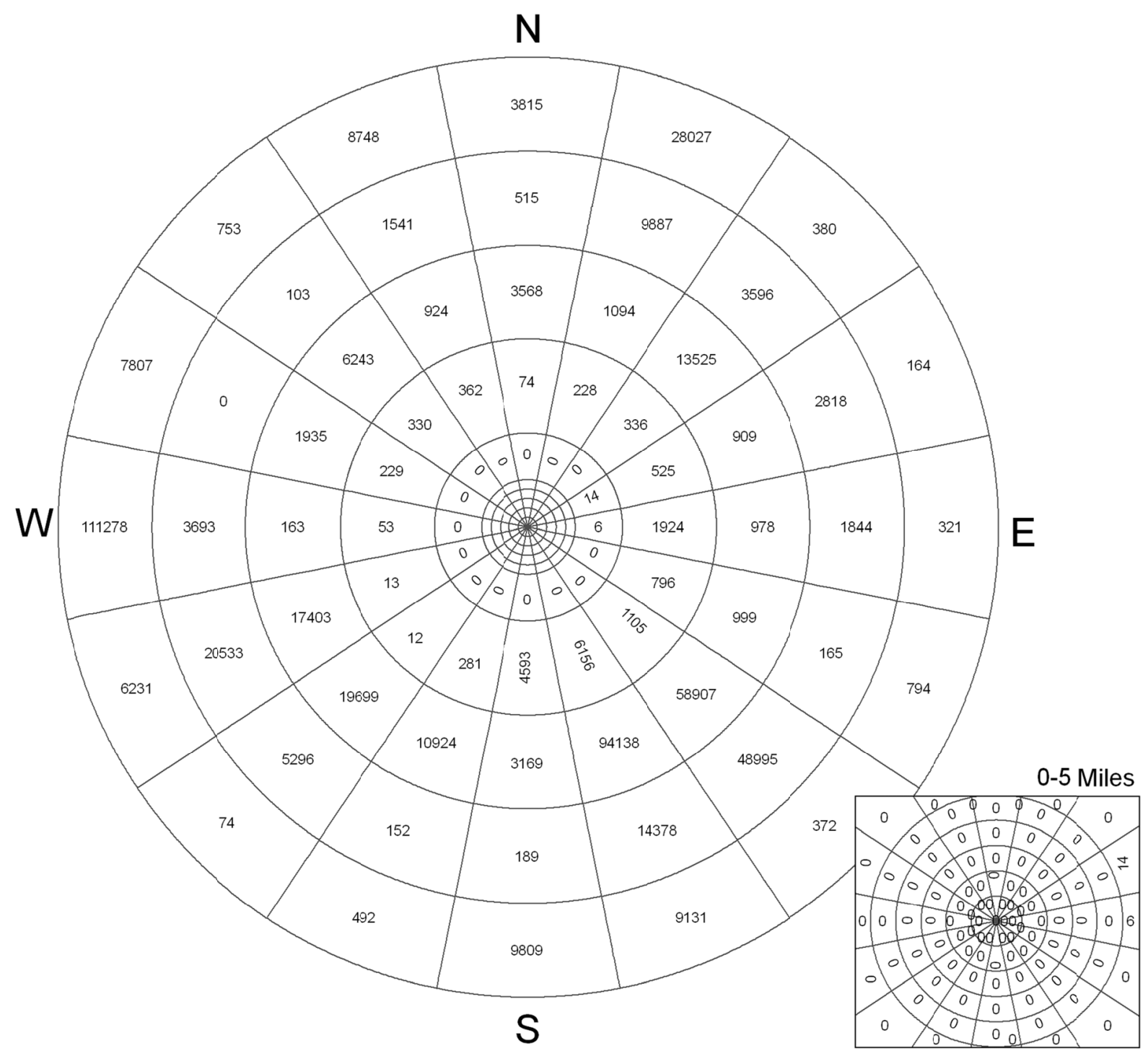

Figure 3.5. WTP 50-mile Population 
Table 3.1. 100K 50-mile Population

Direction

\begin{tabular}{|c|c|c|c|c|c|c|c|c|c|c|c|c|c|c|c|c|c|}
\hline $\begin{array}{l}\text { Distance } \\
\text { (mi) }\end{array}$ & $\mathrm{N}$ & NNE & $\mathrm{NE}$ & ENE & E & ESE & SE & SSE & $\mathrm{S}$ & SSW & SW & WSW & W & WNW & NW & NNW & Total \\
\hline $0-1$ & 0 & 0 & 0 & 0 & 0 & 0 & 0 & 0 & 0 & 0 & 0 & 0 & 0 & 0 & 0 & 0 & 0 \\
\hline $1-2$ & 0 & 0 & 0 & 0 & 0 & 0 & 0 & 0 & 0 & 0 & 0 & 0 & 0 & 0 & 0 & 0 & 0 \\
\hline $2-3$ & 0 & 0 & 0 & 0 & 0 & 0 & 0 & 0 & 0 & 0 & 0 & 0 & 0 & 0 & 0 & 0 & 0 \\
\hline $3-4$ & 0 & 0 & 0 & 0 & 0 & 0 & 0 & 0 & 0 & 0 & 0 & 0 & 0 & 0 & 0 & 0 & 0 \\
\hline $4-5$ & 0 & 0 & 0 & 0 & 0 & 0 & 0 & 0 & 0 & 0 & 0 & 0 & 0 & 0 & 0 & 0 & 0 \\
\hline $5-10$ & 25 & 9 & 5 & 0 & 0 & 0 & 0 & 0 & 0 & 0 & 0 & 0 & 7 & 58 & 250 & 117 & 471 \\
\hline $10-20$ & 2950 & 911 & 450 & 1168 & 494 & 661 & 47 & 0 & 2 & 5 & 8 & 51 & 1605 & 6313 & 143 & 401 & 15209 \\
\hline $20-30$ & 364 & 803 & 661 & 12769 & 672 & 2789 & 2324 & 40313 & 7536 & 2339 & 2466 & 241 & 2 & 3 & 809 & 851 & 74942 \\
\hline $30-40$ & 1600 & 37265 & 4234 & 217 & 4515 & 224 & 48657 & 121509 & 168 & 23926 & 30424 & 14627 & 6716 & 122 & 146 & 2164 & 296514 \\
\hline $40-50$ & 11552 & 1401 & 297 & 132 & 359 & 768 & 9494 & 3401 & 274 & 126 & 172 & 21074 & 129414 & 5480 & 1104 & 8388 & 193436 \\
\hline Total & 16491 & 40389 & 5647 & 14286 & 6040 & 4442 & 60522 & 165223 & 7980 & 26396 & 33070 & 35993 & 137744 & 11976 & 2452 & 11921 & 580572 \\
\hline
\end{tabular}


Table 3.2. 200 HMS 50-mile Population

\begin{tabular}{|c|c|c|c|c|c|c|c|c|c|c|c|c|c|c|c|c|c|}
\hline \multicolumn{18}{|c|}{ Direction } \\
\hline $\begin{array}{l}\text { Distance } \\
\text { (mi) }\end{array}$ & $\mathrm{N}$ & NNE & $\mathrm{NE}$ & ENE & E & ESE & SE & SSE & $\mathrm{S}$ & SSW & SW & WSW & W & WNW & NW & NNW & Total \\
\hline $0-1$ & 0 & 0 & 0 & 0 & 0 & 0 & 0 & 0 & 0 & 0 & 0 & 0 & 0 & 0 & 0 & 0 & $\mathbf{0}$ \\
\hline $1-2$ & 0 & 0 & 0 & 0 & 0 & 0 & 0 & 0 & 0 & 0 & 0 & 0 & 0 & 0 & 0 & 0 & 0 \\
\hline $2-3$ & 0 & 0 & 0 & 0 & 0 & 0 & 0 & 0 & 0 & 0 & 0 & 0 & 0 & 0 & 0 & 0 & $\mathbf{0}$ \\
\hline $3-4$ & 0 & 0 & 0 & 0 & 0 & 0 & 0 & 0 & 0 & 0 & 0 & 0 & 0 & 0 & 0 & 0 & $\mathbf{0}$ \\
\hline $4-5$ & 0 & 0 & 0 & 0 & 0 & 0 & 0 & 0 & 0 & 0 & 0 & 0 & 0 & 0 & 0 & 0 & $\mathbf{0}$ \\
\hline $5-10$ & 0 & 0 & 0 & 0 & 0 & 0 & 0 & 0 & 0 & 0 & 0 & 0 & 12 & 0 & 2 & 1 & 15 \\
\hline $10-20$ & 187 & 39 & 67 & 271 & 501 & 316 & 97 & 2377 & 1093 & 156 & 28 & 41 & 56 & 1675 & 5757 & 359 & 13020 \\
\hline $20-30$ & 3405 & 1504 & 13001 & 1513 & 2544 & 1777 & 66411 & 40040 & 1143 & 13012 & 35869 & 3475 & 289 & 0 & 1193 & 659 & 185835 \\
\hline $30-40$ & 425 & 5977 & 3903 & 1859 & 3119 & 402 & 115766 & 3451 & 188 & 196 & 3353 & 23863 & 15182 & 4 & 29 & 1626 & 179343 \\
\hline $40-50$ & 3355 & 32951 & 803 & 153 & 288 & 971 & 1261 & 9387 & 4512 & 287 & 80 & 3243 & 127411 & 11199 & 3656 & 8730 & 208287 \\
\hline Total & 7372 & 40471 & 17774 & 3796 & 6452 & 3466 & 183535 & 55255 & 6936 & 13651 & 39330 & 30622 & 142950 & 12878 & 10637 & 11375 & 586500 \\
\hline
\end{tabular}


Table 3.3. 300 Area 50-mile Population

Direction

\begin{tabular}{|c|c|c|c|c|c|c|c|c|c|c|c|c|c|c|c|c|c|}
\hline $\begin{array}{l}\text { Distance } \\
(\mathrm{mi})\end{array}$ & $\mathrm{N}$ & NNE & $\mathrm{NE}$ & ENE & $\mathrm{E}$ & ESE & SE & SSE & $\mathrm{S}$ & SSW & SW & WSW & $\mathrm{W}$ & WNW & $\mathrm{NW}$ & NNW & Total \\
\hline $0-1$ & 0 & 0 & 1 & 1 & 0 & 0 & 0 & 0 & 0 & 0 & 0 & 0 & 0 & 0 & 0 & 0 & 2 \\
\hline $1-2$ & 0 & 0 & 2 & 8 & 29 & 24 & 18 & 22 & 1 & 1 & 0 & 0 & 0 & 0 & 0 & 0 & 105 \\
\hline $2-3$ & 0 & 1 & 32 & 57 & 27 & 39 & 112 & 466 & 246 & 4 & 1 & 0 & 0 & 0 & 0 & 0 & 985 \\
\hline $3-4$ & 0 & 5 & 35 & 123 & 44 & 39 & 100 & 1317 & 3261 & 215 & 1117 & 48 & 0 & 0 & 0 & 0 & 6304 \\
\hline $4-5$ & 0 & 24 & 37 & 78 & 98 & 74 & 40 & 177 & 8204 & 133 & 136 & 26 & 4 & 0 & 0 & 0 & 9031 \\
\hline $5-10$ & 4 & 236 & 325 & 414 & 310 & 130 & 15107 & 14896 & 28773 & 11526 & 4325 & 890 & 432 & 0 & 0 & 0 & 77368 \\
\hline $10-20$ & 247 & 2020 & 885 & 505 & 186 & 656 & 50970 & 67443 & 10485 & 981 & 2321 & 5117 & 161 & 1 & 1 & 2 & 141981 \\
\hline $20-30$ & 1513 & 1667 & 3573 & 53 & 682 & 361 & 956 & 68 & 301 & 80 & 106 & 13571 & 937 & 40 & 2 & 12 & 23922 \\
\hline $30-40$ & 4787 & 8135 & 1624 & 278 & 185 & 100 & 965 & 65 & 33838 & 4208 & 86 & 14711 & 25767 & 127 & 7646 & 1153 & 103675 \\
\hline $40-50$ & 2661 & 3655 & 169 & 124 & 483 & 23994 & 1819 & 252 & 2595 & 4131 & 335 & 102 & 22823 & 463 & 1360 & 3778 & 68744 \\
\hline Total & 9212 & 15743 & 6683 & 1641 & 2044 & 25417 & 70087 & 84706 & 87704 & 21279 & 8427 & 34465 & 50124 & 631 & 9009 & 4945 & 432117 \\
\hline
\end{tabular}


Table 3.4. 400 Area 50-mile Population

Direction

\begin{tabular}{|c|c|c|c|c|c|c|c|c|c|c|c|c|c|c|c|c|c|}
\hline $\begin{array}{c}\text { Distance } \\
(\mathrm{mi})\end{array}$ & $\mathrm{N}$ & NNE & $\mathrm{NE}$ & ENE & E & ESE & SE & SSE & $\mathrm{S}$ & SSW & SW & WSW & $\mathrm{W}$ & WNW & NW & NNW & Total \\
\hline $0-1$ & 0 & 0 & 0 & 0 & 0 & 0 & 0 & 0 & 0 & 0 & 0 & 0 & 0 & 0 & 0 & 0 & 0 \\
\hline $1-2$ & 0 & 0 & 0 & 0 & 0 & 0 & 0 & 0 & 0 & 0 & 0 & 0 & 0 & 0 & 0 & 0 & 0 \\
\hline $2-3$ & 0 & 0 & 0 & 0 & 0 & 0 & 0 & 0 & 0 & 0 & 0 & 0 & 0 & 0 & 0 & 0 & 0 \\
\hline $3-4$ & 0 & 0 & 0 & 0 & 0 & 0 & 0 & 0 & 0 & 0 & 0 & 0 & 0 & 0 & 0 & 0 & 0 \\
\hline $4-5$ & 0 & 0 & 0 & 0 & 2 & 0 & 0 & 0 & 0 & 54 & 58 & 0 & 0 & 0 & 0 & 0 & 114 \\
\hline $5-10$ & 1 & 17 & 143 & 236 & 304 & 640 & 572 & 12129 & 5960 & 1086 & 246 & 4 & 0 & 0 & 0 & 0 & 21338 \\
\hline $10-20$ & 55 & 481 & 1909 & 1021 & 822 & 584 & 57756 & 103405 & 15819 & 5190 & 3093 & 270 & 7 & 0 & 1 & 1 & 190414 \\
\hline $20-30$ & 543 & 13484 & 953 & 2915 & 70 & 661 & 14004 & 11073 & 91 & 188 & 8095 & 17653 & 418 & 72 & 502 & 262 & 70984 \\
\hline $30-40$ & 1732 & 2895 & 1419 & 131 & 201 & 645 & 294 & 281 & 15138 & 3253 & 118 & 18753 & 12567 & 147 & 8320 & 3708 & 69602 \\
\hline $40-50$ & 28029 & 2546 & 124 & 356 & 53 & 190 & 1499 & 288 & 22077 & 4324 & 206 & 216 & 23782 & 5241 & 222 & 1140 & 90293 \\
\hline Total & 30360 & 19423 & 4548 & 4659 & 1452 & 2720 & 74125 & 127176 & 59085 & 14095 & 11816 & 36896 & 36774 & 5460 & 9045 & 5111 & 442745 \\
\hline
\end{tabular}


Table 3.5. WTP 50-mile Population

\begin{tabular}{|c|c|c|c|c|c|c|c|c|c|c|c|c|c|c|c|c|c|}
\hline \multicolumn{18}{|c|}{ Direction } \\
\hline $\begin{array}{l}\text { Distance } \\
\text { (mi) }\end{array}$ & $\mathrm{N}$ & NNE & NE & ENE & $\mathrm{E}$ & ESE & SE & SSE & $\mathrm{S}$ & SSW & SW & WSW & W & WNW & NW & NNW & Total \\
\hline $0-1$ & 0 & 0 & 0 & 0 & 0 & 0 & 0 & 0 & 0 & 0 & 0 & 0 & 0 & 0 & 0 & 0 & $\mathbf{0}$ \\
\hline $1-2$ & 0 & 0 & 0 & 0 & 0 & 0 & 0 & 0 & 0 & 0 & 0 & 0 & 0 & 0 & 0 & 0 & $\mathbf{0}$ \\
\hline $2-3$ & 0 & 0 & 0 & 0 & 0 & 0 & 0 & 0 & 0 & 0 & 0 & 0 & 0 & 0 & 0 & 0 & $\mathbf{0}$ \\
\hline $3-4$ & 0 & 0 & 0 & 0 & 0 & 0 & 0 & 0 & 0 & 0 & 0 & 0 & 0 & 0 & 0 & 0 & $\mathbf{0}$ \\
\hline $4-5$ & 0 & 0 & 0 & 0 & 0 & 0 & 0 & 0 & 0 & 0 & 0 & 0 & 0 & 0 & 0 & 0 & $\mathbf{0}$ \\
\hline $5-10$ & 0 & 0 & 0 & 14 & 6 & 0 & 0 & 0 & 0 & 0 & 0 & 0 & 0 & 0 & 0 & 0 & 20 \\
\hline $10-20$ & 74 & 228 & 336 & 525 & 1924 & 796 & 1105 & 6156 & 4593 & 281 & 12 & 13 & 53 & 229 & 330 & 362 & 17017 \\
\hline $20-30$ & 3568 & 1094 & 13525 & 909 & 978 & 999 & 58907 & 94138 & 3169 & 10924 & 19699 & 17403 & 163 & 1935 & 6243 & 924 & 234578 \\
\hline $30-40$ & 515 & 9887 & 3596 & 2818 & 1844 & 165 & 48995 & 14378 & 189 & 152 & 5296 & 20533 & 3693 & 0 & 103 & 1541 & 113705 \\
\hline $40-50$ & 3815 & 28027 & 380 & 164 & 321 & 794 & 372 & 9131 & 9809 & 492 & 74 & 6231 & 111278 & 7807 & 753 & 8748 & 188196 \\
\hline Total & 7972 & 39236 & 17837 & 4430 & 5073 & 2754 & 109379 & 123803 & 17760 & 11849 & 25081 & 44180 & 115187 & 9971 & 7429 & 11575 & 553516 \\
\hline
\end{tabular}




\subsection{Notes on Data Use}

The data presented in this report strictly follow the evaluation procedure described, and no additional verification has been performed. The process of averaging populations over the census block area may indicate the presence of a residential individual where none exists, or no individuals where one does exist. In addition, census data may not be complete for some census blocks due to collection issues within the U.S. Census. These are important factors to consider, especially for dose calculations at distances in closer proximity to an emission source. 



\subsection{Multi-year Population Summary}

The 50-mile populations have been evaluated from 1990 (Beck et al. 1991), 2000 (Elliott et al. 2004), and the current 2010 U.S. Census data. Table 5.1 summarizes these total population data for the operational areas evaluated. The operational areas evaluated changed over time to reflect the locations of major radiological activities. As indicated in the table, the population change continues an upward trend and has remained rather stable (about 20-30\%) over each decade. Year 2010 was the first census year that a 50-mile population of the Hanford Site exceeded the half-million mark for any operational area centroid location.

Table 5.1. 50-mile Populations - 1990, 2000, and 2010 Census'

\begin{tabular}{|c|c|c|c|c|c|}
\hline Centroid & $\begin{array}{c}1990 \\
\text { Census }\end{array}$ & $\begin{array}{l}2000 \\
\text { Census }\end{array}$ & $\begin{array}{l}2010 \\
\text { Census }\end{array}$ & $\begin{array}{l}\text { Year } 1990 \text { to } 2000 \\
\text { Change }\end{array}$ & $\begin{array}{c}\text { Year } 2000 \text { to } 2010 \\
\text { Change }\end{array}$ \\
\hline $100 \mathrm{~F}$ & 303,022 & 382,206 & N/A & $26 \%$ increase & $\mathrm{N} / \mathrm{A}$ \\
\hline $100 \mathrm{~K}$ & N/A & 481,857 & 580,572 & N/A & $20 \%$ increase \\
\hline $100 \mathrm{~N}$ & 375,249 & N/A & N/A & N/A & N/A \\
\hline 200HMS & 375,860 & 486,294 & 586,500 & $29 \%$ increase & $21 \%$ increase \\
\hline 200W (PFP) & 381,936 & N/A & N/A & N/A & N/A \\
\hline 300 Area & 281,586 & 349,067 & 432,117 & $24 \%$ increase & $24 \%$ increase \\
\hline 400 Area & 283,228 & 353,712 & 442,745 & $25 \%$ increase & $25 \%$ increase \\
\hline WTP & N/A & N/A & 553,516 & N/A & $\mathrm{N} / \mathrm{A}$ \\
\hline
\end{tabular}





\subsection{References}

Beck DM, MJ Scott, MD Davis, SF Shindle, BA Napier, AG Thurman, DB Pittenger, and NC Batishko. 1991. Hanford Area 1990 Population and 50-year Projections. PNL-7803, Pacific Northwest National Laboratory, Richland, Washington.

Elliott DB, EJ Antonio, and K Rhoads. 2004. Hanford Area 2000 Population. PNNL-14428, Pacific Northwest National Laboratory, Richland, Washington.

ESRI - Environmental Systems Research Institute. 2009. ArcGIS Desktop: Release 9.3.1. Redlands, California.

U.S. Census Bureau (Census). 2010a. “Census 2010 Redistricting Data (P.L. 94-171) Summary File Oregon.” (Website: http://www2.census.gov/census_2010/01-Redistricting_File--PL_94-171/Oregon/). U.S. Census Bureau, Washington, D.C. Accessed July 18, 2011.

U.S. Census Bureau (Census). 2010b. "Census 2010 Redistricting Data (P.L. 94-171) Summary File Washington. (Website: http://www2.census.gov/census_2010/01-Redistricting_File--PL_94171/Washington/). U.S. Census Bureau, Washington, D.C. Accessed July 18, 2011.

U.S. Census Bureau (Census). 2010c. "Census 2010 Block Tiger/Line Shapefiles.” (Website: http://www.census.gov/cgi-bin/geo/shapefiles2010/main). U.S. Census Bureau, Washington, D.C. Accessed July 18, 2011. 



\section{Distribution}

No. of

Copies

DOE Richland Operations Office

DE Jackson

A4-52

DOE Pacific Northwest Site Office

T Aldridge

K9-42

2 Washington State Department of Health

Radioactive Air Emissions Section

PJ Martell, Manager

J Schmidt

B1-42

2 Mission Support Alliance, LLC

DJ Rokkan

H7-28
No. of

Copies

Washington Closure Hanford, LLC

JG Woolard

H4-21

10 Pacific Northwest National Laboratory

EJ Antonio

K3-54

JM Barnett

J2-25

EL Hamilton

K6-52

BA Napier

K3-54

K Rhoads

K3-54

SF Snyder (3)

K3-54

PNNL Reference Library

P8-55 




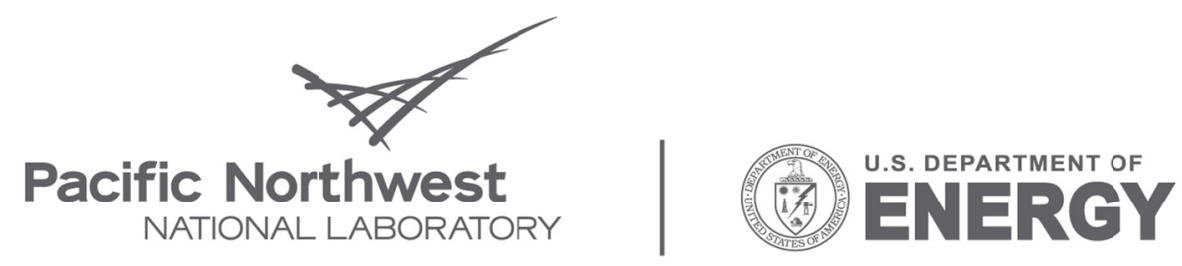

Proudly Operated by Battelle Since 1965

902 Battelle Boulevard

P.O. Box 999

Richland, WA 99352

1-888-375-PNNL (7665)

www.pnl.gov 